\title{
RELATO DE CASO CLÍNICO - SÍNDROME DE GOLDENHAR
}

\section{GOLDENHAR'S SYNDROME - A CASE REPORT}

\author{
Nuno Figueiredo Pinto ${ }^{1}$, Márcia Cançado Figueiredo ${ }^{2}$, \\ Cinthya Aline das Dores Guarienti ${ }^{3}$
}

1 Av. Afonso Pena, 262, ap. 1402, Belo Horizonte, MG

2 Autor para contato: Faculdade de Odontologia da Universidade Federal do Rio Grande do Sul - UFRGS, Departamento de Cirurgia e Ortopedia, Porto Alegre, RS, Brasil; (51)33375471-33255738; e-mail: marciacf@myway.com.br

3 Bebê Clínica da Universidade Federal do Rio Grande do Sul - UFRGS, Porto Alegre, RS

Recebido para publicação em 15/02/2004

Aceito para publicação em 01/06/2005

\section{RESUMO}

A Síndrome de Goldenhar é rara, caracterizada por anomalias congênitas em diferentes locais do corpo, principalmente na face, orelhas e vértebras (Ryan, 1988). Gorlin et al (1963) sugeriram o termo displasia Oculo-Auriculo-Vertebral (OAV) incluindo anomalias vertebrais nessa síndrome. A sua etiologia é pouco clara, apresentando-se geneticamente variável e de causa bastante heterogênea. O presente trabalho descreve essa síndrome e suas características predominantes, baseadas na revisão da literatura atual, bem como, nas observações clínicas durante o tratamento ortodôntico realizado ao longo de onze anos de um paciente do sexo masculino portador de características clássicas da mesma. É de suma importância diagnosticar e intervir precocemente, o que contribuirá significantemente para a obtenção de sucesso, melhorando as condições do paciente afetado.

Palavras-chave: Síndrome de Goldenhar, maloclusão, tatamento ortodôntico

\begin{abstract}
Goldenhar's syndrome is a rare condition described initially in the early 1950's. It is characterized by a combination of anomalies: dermal epibulbar cysts, auricular appendices and malformation of the ears (Ryan, 1988). Gorlin suggested the name oculo-auriculo-vertebral (OAV) dysplasia for this condition and also included vertebral anomalies as signs of the syndrome. The etiology of this rare disease is not fully understood, as it has shown itself variable genetically and of unclear causes. This work reports a case of Goldenhar's syndrome in a male who
\end{abstract}


presented all classical signs of this rare syndrome and received orthodontic treatment during eleven years. It is very important to diagnose it as early as possible, to obtain a success in the treatment, and to improve the conditions of the patient.

Key words: Goldenhar's syndrome, malocclusion, orthodontic treatment

\section{Introdução}

A Síndrome de Goldenhar é uma anomalia congênita rara, de etiologia ainda desconhecida e caracterizada por uma tríade clássica de alterações oculares, auriculares e vertebrais (Brosco et al., 2004).

Vários termos têm sido usados para denominar essa entidade: microssomia hemifacial, displasia oculoauriculovertebral, síndrome de Goldenhar-Gorlin, síndrome do primeiro e segundo arcos branquiais e displasia facial lateral. Aceita-se que o termo espectro óculo-auriculo-vertebral seja o mais correto. É causada por uma herança multifatorial, apresentando história familiar positiva em 6\% dos casos (Bueno, 2004). Os principais defeitos encontrados nesse conjunto de malformações são devidos principalmente a erros de morfogênese do primeiro e segundo arcos branquiais, acompanhados em alguns casos de malformações das vértebras e/ou dos olhos (Silva e Penido, 2003).

Em 1881 o primeiro caso foi descrito por Von Arit (1973). A síndrome foi caracterizada por Goldenhar (1952/1953), em detalhe, recebendo seu nome, em 1963. Gorlin et al. em 1963, sugerem o nome síndrome óculoaurículovertebral (OAV) incluindo anomalias vertebrais como características dessa síndrome (Pinheiro et al., 2003). Nas descrições clássicas são relatadas a presença de dermólide epibulbar, apêndices pré-auriculares, hipoplasia de mandíbula e anomalias vertebrais (Bustamante et al., 1989).

\section{Caso Clínico}

O paciente A.G.A.S., nascido em 14 de abril de 1967 em Minas Gerais, foi encaminhado ao consultório dentário de um ortodontista por um cirurgião bucomaxilofacial, que, juntamente com uma equipe de médicos cirurgiões plásticos estava acompanhando esse paciente desde o seu nascimento, e já haviam realizado algumas correções cirúrgicas necessárias. A.G.A.S, que na época apresentava-se com 8 anos de idade permaneceu sob tratamento/acompanhamento com essa equipe multiprofissional durante 11 anos, ou seja, de 1975 a 1986.

Interessante ressaltar que, avaliando-se o relatório histórico desse paciente, o mesmo não apresenta antecedentes familiares de portadores dessa síndrome. Quanto às suas características clínicas, estas serão ilustradas e mencionadas na discussão do caso.

Muitas dificuldades foram encontradas no decorrer do tratamento devido à complexidade do caso, a falta de uma literatura específica com relação à abordagem ortodôntica, bem como casuísticas que pudessem espelhar a conduta clínico-cirúrgica a ser realizada. O paciente era mensalmente avaliado, quando não estava internado devido às inúmeras cirurgias plásticas às quais foi submetido.

A aparatologia ortodôntica constituiu-se da técnica fixa de "edgewise” associada a placas ortopédicas específicas para o caso, objetivando não só a expansão da atresia maxilar como o estímulo miofacial. Devido à hipoplasia mandibular, utilizou-se, inicialmente, o monobloco convencional de Andreasen, como o ativador neuro-muscular de crescimento (Hanson e Barret, 1995).

\section{Discussão}

A incidência da Síndrome de Goldenhar situa-se entre 1 em 3500 e 1 em 26000 nascimentos. Esta síndrometem geralmente uma causa multifatorial. Poswillo, em 1974, apresenta a incidência de 1/3500 nascimentos. Complexa, constitui um contínuo “spectrum”. Há cons- 
tatação em sucessivas gerações com causas como consangüinidade ou fatores genéticos, com autossoma dominante, recessivo ou multifatorial. Regenbogen et al. (1982), relatou que a morfologia da crista neural seria afetada, o que ocasionaria malformação dos derivados do primeiro e segundo arcos branquiais, afetando a face, orelhas, olhos, vértebras e causando outras anomalias. Citando textualmente Gorlin et al. (1963): "Notamos que a severidade de displasias oriundas do 1 e 2 arcos branquiais, podem apresentar formas “sui generis", não consideradas específicas dos complexos sintomas, em que a patogênese pode ser severa e de diferentes etiologias. Alguns podem ter extensa linha de anomalias e outros, menores, com anomalias semelhantes”.

As complicações da Síndrome de Goldenhar decorrem sobretudo da presença de anomalias crâniofaciais. As malformações do ouvido externo encontradas são várias e vão desde simples alterações do pavilhão auricular até a sua ausência completa e do canal auditivo externo. Existe perda da audição em 15\% dos casos. Os olhos e o sistema nervoso central podem também ser afetados. Pode haver anomalias das glândulas salivares e hipoplasia da língua. Dentro das anomalias do esqueleto, as mais freqüentes são as da coluna vertebral, nomeadamente a fusão das vértebras cervicais que pode ser encontrada em cerca de 20$35 \%$ dos doentes. Pode-se também encontrar anomalias cardíacas, pulmonares, renais e gastrointestinais. As características peculiares dessa síndrome estão descritas a seguir, sendo que aquelas que foram observadas no paciente em questão estão ilustradas abaixo:

\subsection{Olhos}

Stanojevic et al, em 2000, relataram a presença de anormalidades nos olhos, que podem apresentarse como pequenas manchas dermóides e lipodermóides epibulbares. Segundo Baum et al (1973), ela apresenta coloboma, geralmente na parte superior dos olhos, Freqüentemente a fissura palpebral é mais descendente. Pode ocorrer uma obstrução nasolacrimal e oftalmoplasia (paralisia ocular) ou síndrome de Dnam: a pálpebra fecha um olho, quando olha de lado com atresia e hipersensi-bilidade corneal (Nema,1973). Catarata atrofiada, enoftalmia (retração anormal do olho dentro da órbita).Cistos dermóides e fístulas secas (Nema,1973).
Os dermóides podem estar localizados na marginal da córnea, no quadrante inferior externo. Alguns pacientes têm dermóides elipodermóides idênticos nos olhos. Nessas condições são bilateriais. Inclinações antimongol da fissura palpebral com fissão no fundo da visão.(Figura 1) Colobomas unilaterais na pálpebra su-perior, atingindo o temporal direito, ocorrendo de 50 a $60 \%$ com os dermóides epibulbares ou lipodermóides, mais em um olho, localizados na junção da esclerótica ou no quadrante inferior. Os lipodermóides são mais freqüentes no quadrante superior do temporal, e o coloboma, na íris (Nema, 1973) Gorlin cita, em 1963 que "quando a microftalmia ou anoftalmia está presente, o retardamento mental ocorreria concomitantemente"

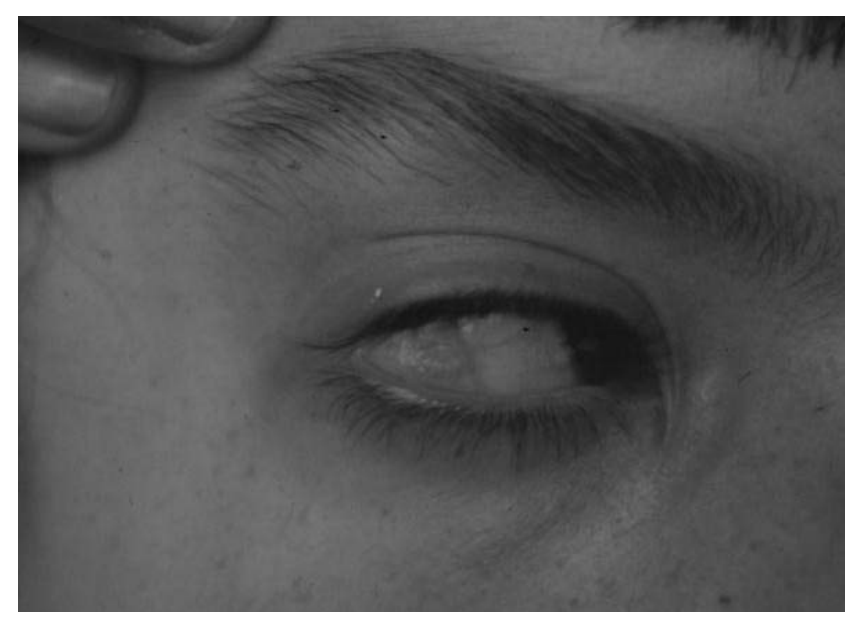

Figura 1 - Paciente A.G.A.S. apresenta anormalidade ocular ou seja, pequenas manchas dermóides e lipodermóides epibulbares: Coloboma na parede lateral superior dos olhos.

\subsection{Orelhas}

As malformações auriculares podem variar da completa aplasia até deformidades no ouvido externo, médio e interno, resultando em perda da audição (Carvalho, et al., 1999). Freqüentemente existem apêndices fibromatosos pré-auriculares (skin tags) e cavidades na frente da orelha ou na linha entre esta e o canto da boca. Nas malformações auriculares, o canal da orelha pode estar completamente ausente, implicando em surdez (unilateral) em aproximadamente $40 \%$ dos casos (Smith, 1989). Segundo Brosco, et al., durante avaliação audiológica de pacientes portadores da síndrome de Goldenhar, em 2004, dos pacientes que possuíam malformação auricular unilateral, apenas 6\% 
tinha comprometimento auditivo na orelha contralateral, sendo 3\% do tipo misto de grau severo e 3\% de grau moderado. (Figura 2)

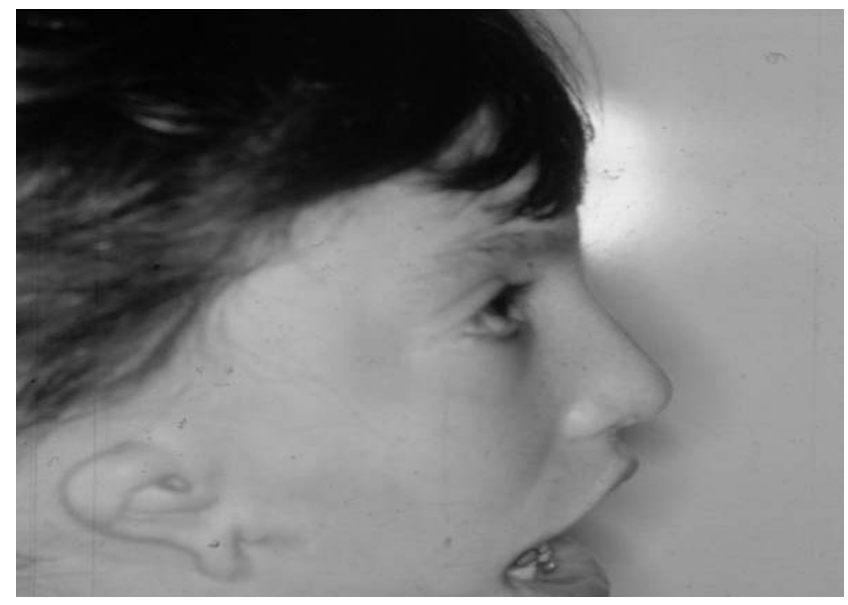

Figura 2 - Presença de malformação auricular.

\subsection{Alterações esqueletais}

A identificação de anomalias na coluna vertebral levou à complementação da síndrome por Gorlin. 40 a $60 \%$ dos pacientes apresentaram vértebras cuneiformes, espinha bífida, escoliose (curvatura lateral anormal da coluna vertebral), sinostoses, com a fusão de várias vértebras ou semi-vértebras. $\mathrm{O}$ pé equinovaro apareceu em 20\% dos casos e, em alguns casos, outras anomalias periféricas esqueletais (Gorlin, 1963; Geeraets, 1976).

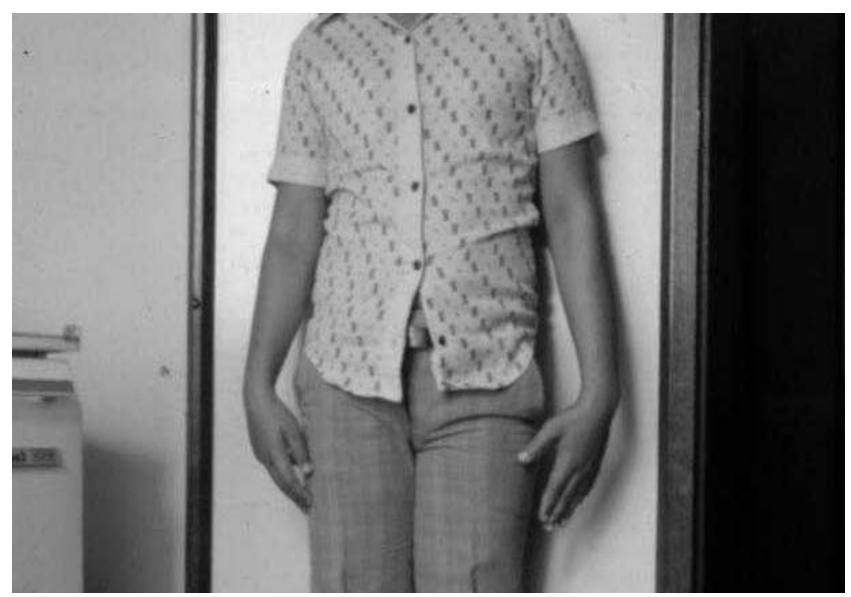

Figura 3 - Paciente A.G.A.S. apresenta escoliose (curvatura lateral anormal da coluna vertebral).

\subsection{Coração}

Smith (1989) relatou que as crianças com a síndrome de Goldenhar podem apresentar problemas cardíacos (comunicação intraventricular, tetralogia de Fallot e coarctação da aorta). Comprometimentos da veia cava e sub-cava esquerda, também são observados, refletindo esse quando nosológico nas alterações cardio-vasculares (Gorlin, 1963; Geeraets, 1976; Bustamante, 1989).

Lam (2000), sugeriu que essa síndrome pode advir de um acidente vascular no feto. Esse acidente causa a diminuição do aporte sanguíneo e a produção de coágulos na área daqueles tecidos que desenvolverão as estruturas da orelha e da mandíbula. A severidade do dano causado pelo acidente vascular estaria diretamente relacionada ao período de desenvolvimento em que este aconteceu, e também à área de destruição que houve no tecido afetado (Silva e Penido, 2003).

\subsection{Face}

A face apresenta, no conjunto, características visuais peculiares a essa síndrome, devido à assimetria facial, hipoplasia e deslocamento do pavilhão auricular, bem como do comprometimento do osso malar e temporal, com envolvimento dos maxilares, resultando no seu achatamento devido à aplasia ou hipoplasia do ramo da mandíbula e do côndilo.(Figuras 4 e 5)

Em estudo sobre o perfil dos indivíduos comprometidos pela síndrome de Goldenhar e a freqüência do comprometimento auditivo destes, Brosco et al. em 2004, observou casos de protuberância frontal, 13\% apresentam envolvimento bilateral facial, sendo mais comum e severa de um lado, principalmente o direito, porém estes dados foram considerados estatisticamente insignificantes, bem como a relação da síndrome com o sexo, onde houve predominância para o gênero feminino. Outras características: Hipoplasia do malar, micrognacia, queixo curto (ou ausente), microstomia microfacial (ocasional), semelhante à síndrome de Franceschetti: “face like a fish” (Nema, 1973; Bueno, 2004). Apresenta ainda a microsomia, envolvimento parcial da estrutura da face (osso zigomático e malar) (Nema, 1973). Erroneamente, achavam ser só da face. 

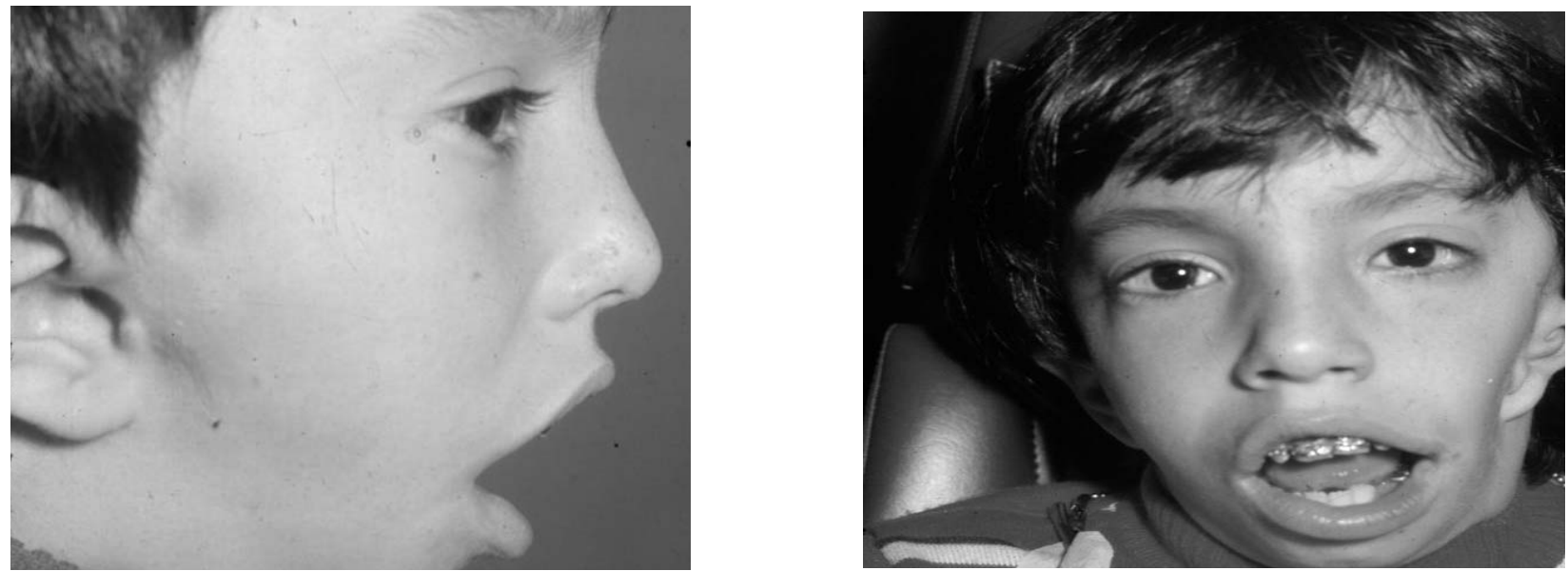

Figuras 4 e 5 - Paciente A.G.A.S. com malformação complexa, unilateral, onde a assimetria facial está presente, os ossos maxilar, temporal e malar reduzidos no tamanho e em espessura.

\subsection{Manifestações bucais}

As manifestações bucais encontradas foram hipoplasia de esmalte, anomalias de forma dentária, ocorrência de supranumerários, língua geográfica, macrostomia, mordida cruzada, mordida profunda e palato profundo (Bueno, 2004), (Figuras 6, 7 e 8). A língua pode ser hipoplásica na musculatura do lado afetado
(Sugar, 1967; Greenwood, et al. 1974). Pode ocorrer uma relativa falência do desenvolvimento neuromuscular e ósseo, devido ao conjunto das hiopoplasias, com a face apresentando debilidade de expressão, geralmente de um lado apenas (Nema, 1973). Também pode ocorrer agenesia da glândula salivar, com aparecimento de fístulas.
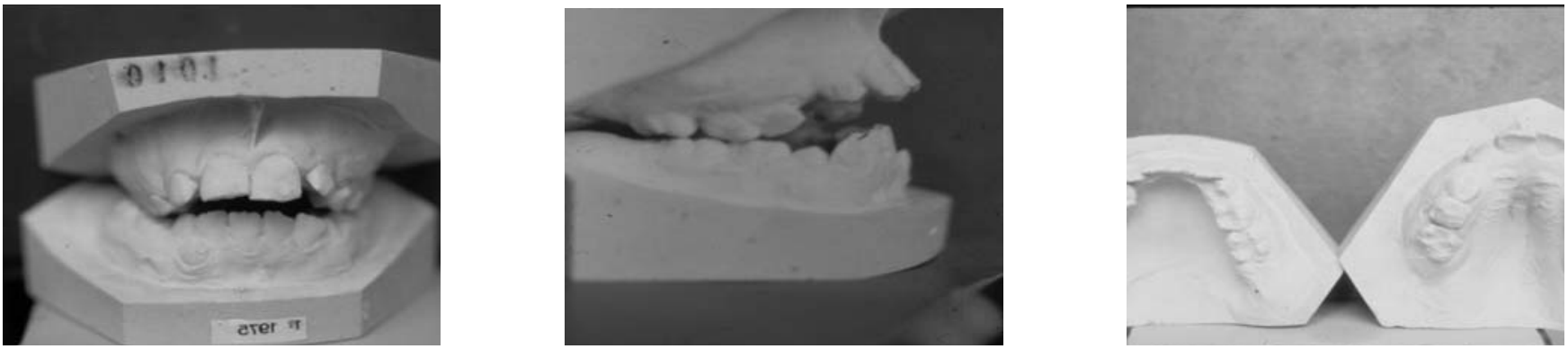

Figuras 6, 7 e 8 - Modelos em gesso iniciais do paciente A.G.A.S. apresentando uma mordida cruzada bilateral, mordida aberta, palato profundo e hipoplasia de esmalte dos primeiros molares permanentes.

O desenvolvimento e a irrupção dos dentes nas duas fases da dentição, decídua ou permanente, pode ocorrer normalmente (Silva e Penido, 2003) (Figuras 9 e 10). 

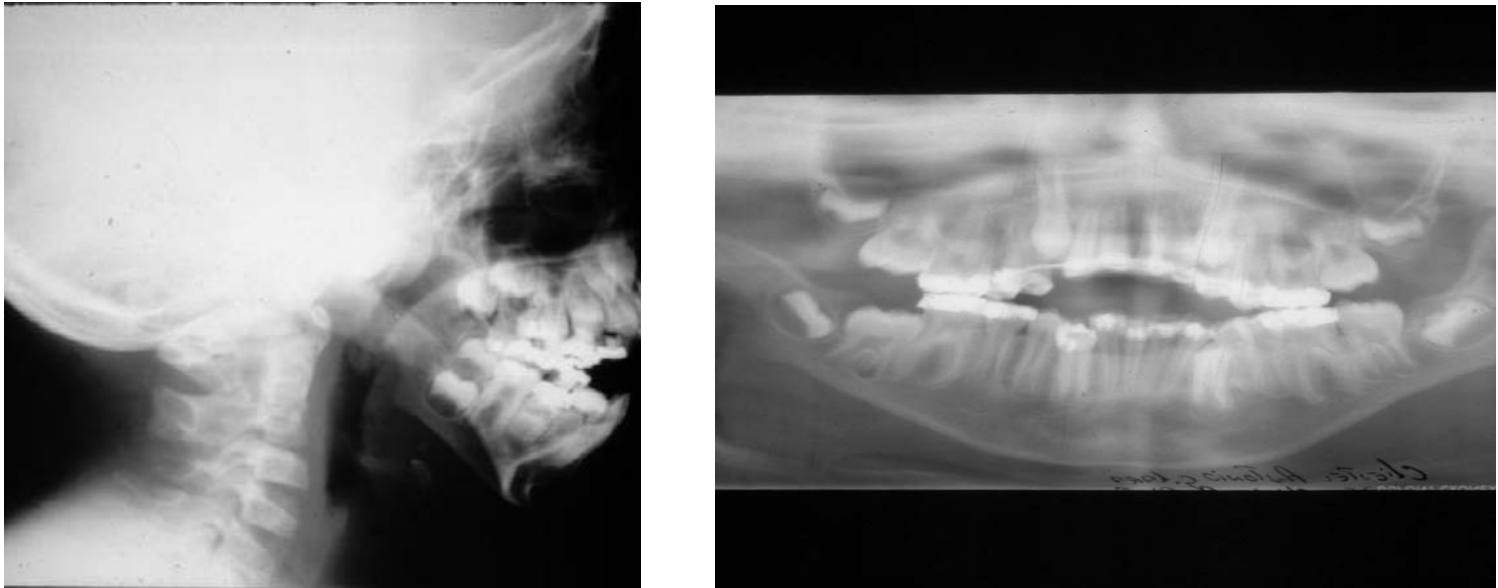

Figuras 9 e 10 - Radiografias lateral e panorâmica iniciais do paciente A.G.A.S certificando a presença de maloclusão instalada e o desenvolvimento e irrupção dos dentes decíduos e permanentes ocorrendo normalmente
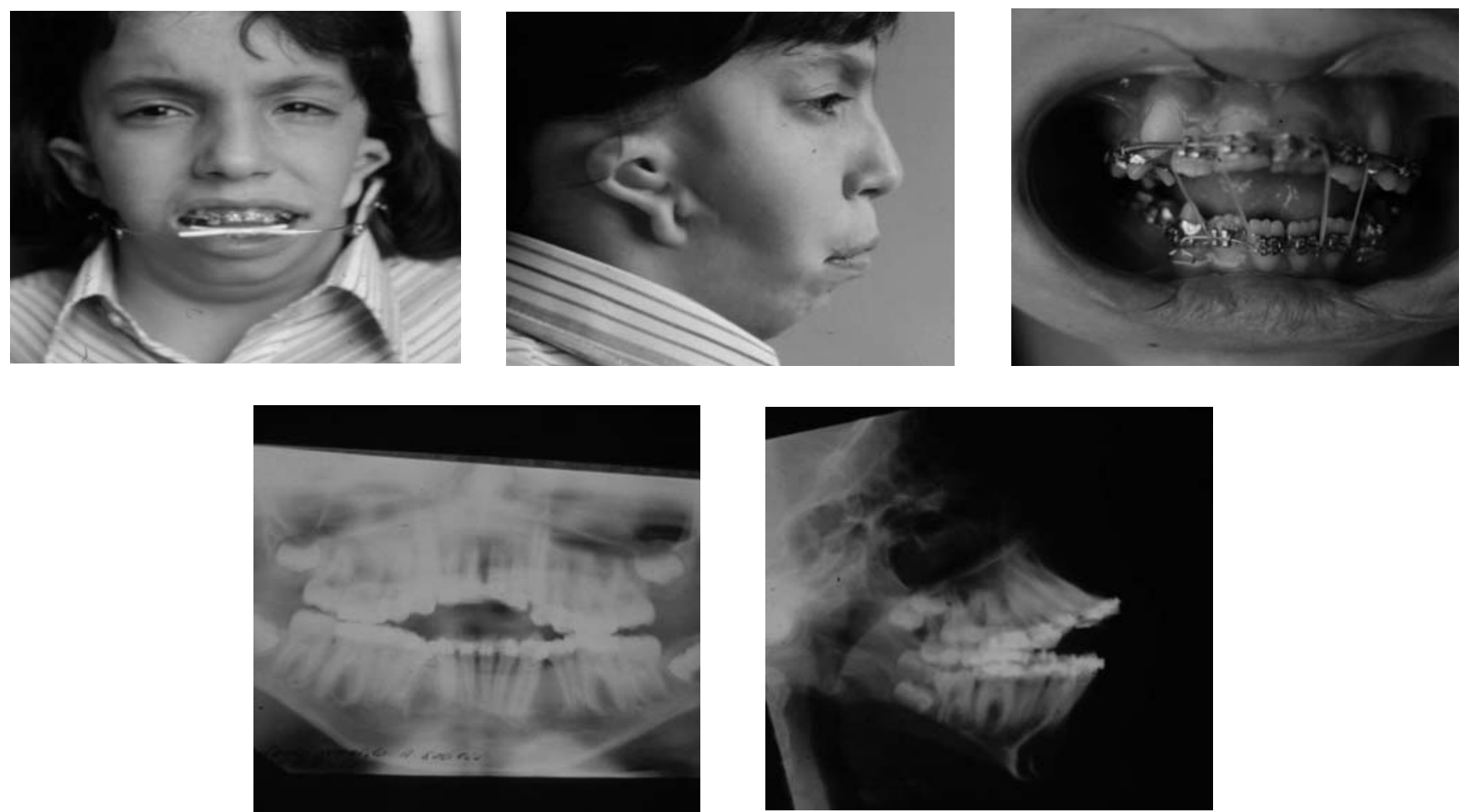

Figuras 11 a 15 - Acompanhamento clinico e radiográfico da terapia ortodôntica do paciente na metade de seu tratamento - 5 anos após, 1980, paciente apresentava-se com 11 anos de idade. 

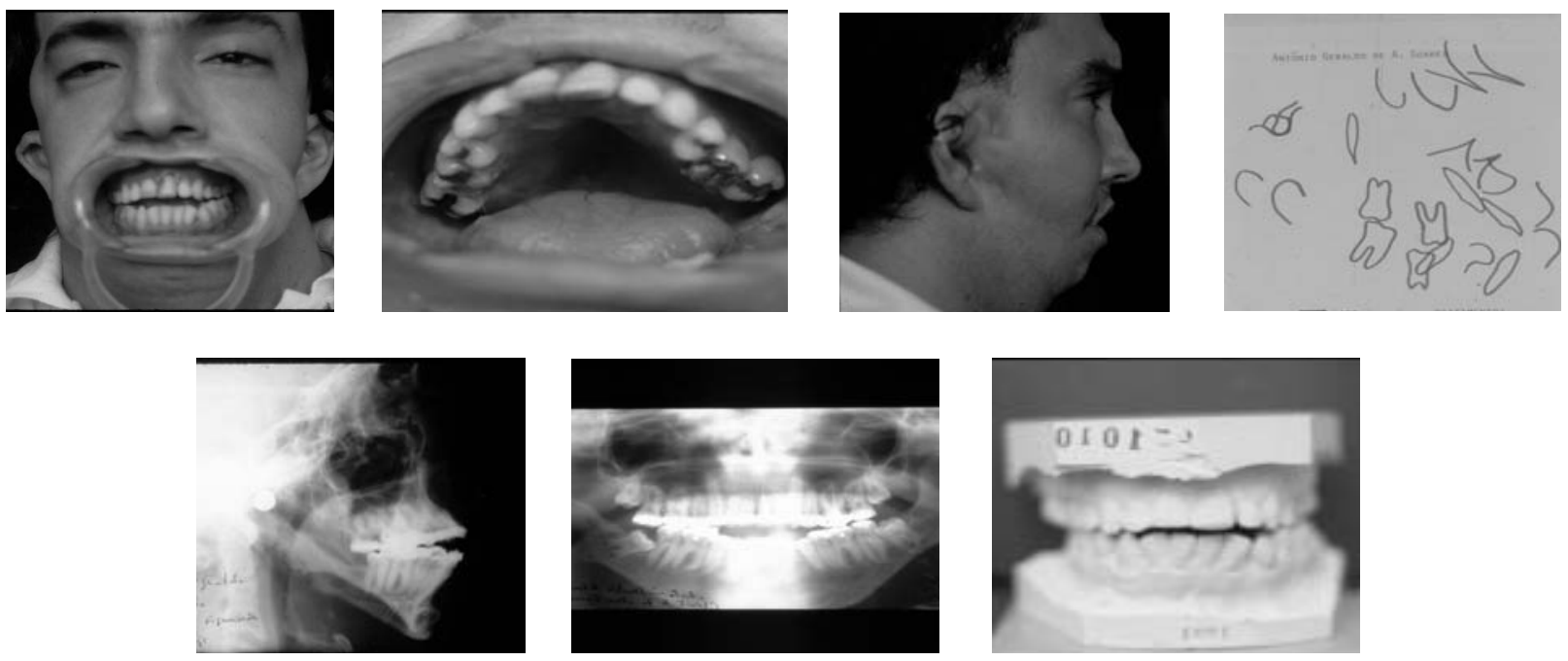

Figuras 16 a 22 - Caso finalizado, aspecto clinico, radiográfico, traçado cefalométrico inicial e após terminada a terapia ortodôntica do paciente, modelos em gesso - 11 anos após , 1986 , paciente apresentava-se com seus 17 anos de idade.

A análise da oclusão dentária pós- tratamento cirúrgico e ortodôntico do paciente A.G.A.S. revela melhora na sua oclusão e simetria facial. Ressalta-se que foi possível finalizar o tratamento ortodôntico, mesmo com dificuldades de manuseio e pouca colaboração do paciente.

\section{Conclusão}

Baseados em nossa experiência, bem como na literatura atual, podemos afirmar que a avaliação cuidadosa da morfologia craniofacial pelo ortodontista é de extrema importância para referenciar precocemente os casos em que seja necessária uma intervenção terapêutica. Para um prognóstico favorável acreditamos que, como parte da avaliação diagnóstica inicial, devem ser feitos vários exames imagiológicos cerebrais, cardíacos, renais e do esqueleto. A avaliação do crescimento da cabeça e do pescoço, da audição e da visão têm especial importância durante a primeira infância, e entre os 3-5 anos é recomendada a realização de radiografias da coluna cervical para a identificação de possíveis fusões das vértebras.

O tratamento deve ser individualmente determinado e a abordagem multiprofissional deve ser considerada, buscando melhorar a aparência e funcionalidade das estruturas acometidas. O controle preventivo posterior no paciente portador da síndrome de Goldenhar é importantíssimo e deve englobar avaliações multidisciplinares, ou seja, uma visão holística, além da dentição, incluíndo o acompanhamento psicológico do paciente e de toda a sua família, a fim de garantir a sua integração na sociedade

\section{REFERÊNCIAS}

1. BAUM, J.L., FEINGOLD, M. Ocular Aspects of Goldenhar's Syndrome. Am. J. Opthalmol, v.75, n.2, p. 250-7, 1973.

2. BROSCO, K.C, ZORZETTO, N.L, COSTA, A.R da, Perfil Audiológico de Indivíduos Portadores da Síndrome de Goldenhar. Ver. Bras. Otorrinolaringol, p70-75, set-out, 2004.

3. BUENO, D.F. Estudo das Alterações Clínicas e Radiográficas da Microssomia Hemifacial. Dissertação de Mestrado. USP/ São Paulo, s.n., 2004, 102p.

4. BUSTAMANTE, L.N.P, GUERRA, I.V. de, IWAHASHI, E.R, EBAID, M, Síndrome de Goldenhar: Relato de 5 casos em Associação com Malformações Cardíacas. Arq. Bras. Cardiol. v.53, n.5, p.287-90, nov.1989.

5. CARVALHO, G.J, SONG C.S, VARGEVIK, K, LALWAKI,A.K, Auditory and facial nerve dysfunction in patients with hemifacial microsomia. Arch Otolaryngol Head Neck Surg. v.125, n.2, and p. 209-12. 1999

6. GEERATS,H.L. apud POSWILLO, D. Otomandibular deformity: pathogenesis as guide to reconstruction,. J. Maxilofacial Surg. v.2, n.3, p.64-72, 1976.

7. GORLIN, R. J., TORIELLO, H. V., COHEN, JR., M. M. 
Hereditary hearing loss and its syndromes. Edit. Oxford University Press. p. 69-72; 120; 193-4, New York, USA 1963.

8. GREENWOOD, F.M. apud REGENBOGEN, L., et al. Further evidence for an autossomal dominant form of oculoauriculovertebral dysplasia. Clin. Genet. V.21, n.3, p.1617,1982.

9. HANSON, M.L., BARRET, R.H. Fundamentos de Miologia Orofacial, Edit. Enelivros, Rio de Janeiro, 1995.

10. LAM, C. H. A theory on the embryogenesis of oculoauriculo-vertebral (Goldenhar) Syndrom. J. Craniofacial Surg., v.11, n.6, p.547-52, 2000.

11. NEMA, H. V. Ophthalmic Syndromes-Edit. Butterworks, USA, 1973.

12. PINHEIRO, A.L.B, ARAÚJO, L.C, OLIVEIRA, S.B, SAMPAIO, M.C.C, FREITAS, A.C, Goldenhar's Syndrome: case report. Braz. Dent. J., v. 14, n.1, p.67-70, 2003.

13. POSWILLO, D. Otomandibular deformity: pathogenesis as guide to reconstruction, J. Maxilofacial Surg. v.2, n.3, p.64$72,1974$.

14. Further evidence for an autossomal dominant form of oculoauriculovertebral dysplasia. Clin. Genet. V.21, n.3, p.1617, 1982.

15. RYAN, C. A., FINNER, N. N., IVES, E. Discordance of signs in monozygotic twins concordant for the Goldenhar anomaly. Am. J. Med. Genet. v.29, n.4, p.755-61, 1988.

16. SILVA, L. C. P. da, PENIDO, C. V. de S. R. Sindrome de Goldenhar: relato de caso clínico. J. Bras. Odontoped. Odontol. Bebe v.6, n.32, p.283-6, 2003.

17. SMITH, D. W. Síndrome de malformações congênitas. Edit.Manole, $3^{\text {a }}$ Ed. São Paulo, 1989.

18. STANOJEVIC, M., STIPOLYEV, F., KOPRCINA, B., KURJAK, A. An oculo-auriculo-vertebral (Goldenhar) spectrum associated with pericentric inversion 9: coincidental findings or etiologic factor? J. Craniofac. Genet. Dev. Biol. v.20, n.3, p.150-4, 2000.

19. SUGAR, P.G. apud RYAN, C. A., FINNER, N. N., IVES, E. Discordance of signs in monozygotic twins concordant for the Goldenhar anomaly. Am. J. Med. Genet. v.29, n.4, p.755-61, 1988.

20. VONARIT, K.V. apud NEMA, H. V. Ophthalmic Syndromes - Edit. Butterworks, USA, 1973 